\title{
Batten disease: a rare case report and review of literature
}

\author{
Deepak Jain*, H K Aggarwal, Pulkit Chhabra, Avinash Rao
}

Department of Medicine, Pt. B.D. Sharma University of Health Sciences, ROHTAK, Haryana, India

\begin{abstract}
Batten disease (also known as Spielmeyer-Vogt-Sjögren-Batten disease) is a rare, fatal autosomal recessive neurodegenerative disorder. It is the most common form of a group of disorders called the neuronal ceroid lipofuscinoses (NCLs). The onset of this disease is between the ages of 5 and 8 years. The typical early signs are progressive vision loss, seizures, ataxia or clumsiness. This disease progresses slowly and ends in death in the late teens or early $20 \mathrm{~s}$, although some may live into their $30 \mathrm{~s}$. We report a case of young male patient, who presented with seizures and mental retardation, diagnosed to be having Batten disease based on clinical and radiological examination.
\end{abstract}

Keywords: vision loss, seizures, mental retardation, optic atrophy

\section{Introduction}

Batten disease is considered the juvenile form of the neuronal ceroid lipofuscinoses (NCLs). It is named after the British pediatrician Frederick Batten, who first described it in 1903 [1]. The NCLs are characterized by abnormal accumulation of certain fatty, granular substances (lipopigments, ceroid and lipofuscin) within nerve cells of the brain as well as other tissues of the body resulting in progressive atrophy of certain areas of the brain along with neurological impairment, and other characteristic symptoms and physical findings. Batten disease is very rare and occurs in an estimated 2 to 4 out of every 100,000 births in the United States. This disease is more prevalent in Finland, Sweden, and other parts of northern Europe, Newfoundland and Canada. The incidence of Batten disease in India is very low and it often affects more than one person in families with the defective genes.

Received: September 2016; Accepted after review: December 2016; Published: December 2016.

${ }^{*}$ Corresponding author: Assist. Prof. Dr. Deepak Jain, Department of Medicine, Pt BDS PGIMS, Rohtak124001, Haryana, India

Email: jaindeepakdr@gmail.com

\section{Case report}

A 21 year old male presented to the emergency room with multiple episodes of uncontrolled seizures and altered behavior for 3 days prior to his admission. There was no history of fever, loss of consciousness, weakness of any of the limbs. Patient was a diagnosed case of mental retardation with seizure disorder since 13 years. He was born through normal vaginal delivery and had normal birth weight. Patient had normal developmental milestones and did his schooling up to the age of 7 years. At the age of 7 years, patient had multiple episodes of generalized tonic clonic type of seizures for which he was put on antiepileptic drugs. There was no history of fever, loss of consciousness or trauma. Radiological examination of the brain was found to be normal. With time, there was progressive deterioration of intelligence, behavioral changes, vision loss and loss of previously acquired physical and mental abilities. There was no past history of any other chronic illness like tuberculosis, asthma, diabetes, trauma or blood transfusion. Family history revealed that his elder brother suffered from similar illness and died at the age of 18 years. 
On general examination, the patient was malnourished, drowsy and afebrile. Vitals were normal. During central nervous system examination patient was conscious but disoriented in time, place and person. Higher mental functions could not be assessed. Cranium and spine were normal. No signs of meningitis were present. Power was $5 / 5$ in all the four limbs. All superficial reflexes and deep tendon reflexes were normal on both the sides of the body. Plantar reflexes were flexor on both sides. The rest of systemic examination including respiratory, cardiovascular and abdominal examination was normal.
On laboratory examination complete hemogram, kidney function tests and liver function test were normal. On microscopy, abnormal white blood cells i.e. vacuolated lymphocytes were seen in peripheral blood smear.

On fundus examination, bilateral optic atrophy was seen with severe attenuated vessels, bony spicules and cystoid macular edema. There were circular bands of different shades of pink and orange at the optic nerve and retina in the back of the eye resembling bull's eye (Figure 1).

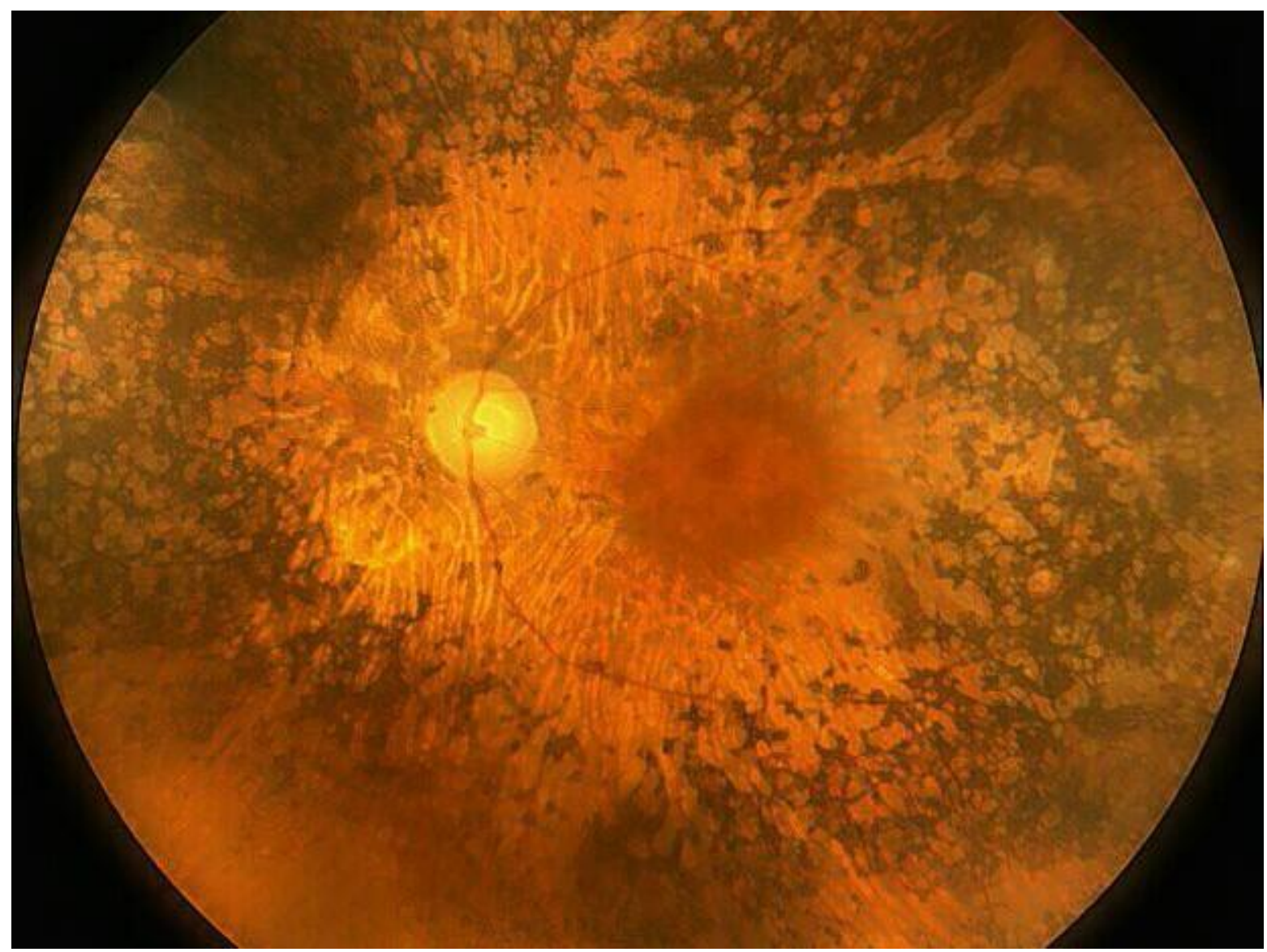

Fig. 1. Fundus examination of patient resembling bull's eye appearance

Electroencephalogram showed spike and wave discharges suggestive of seizure activity.
Brain MRI of the patient at the age of 7 years was normal (Figure 2). 


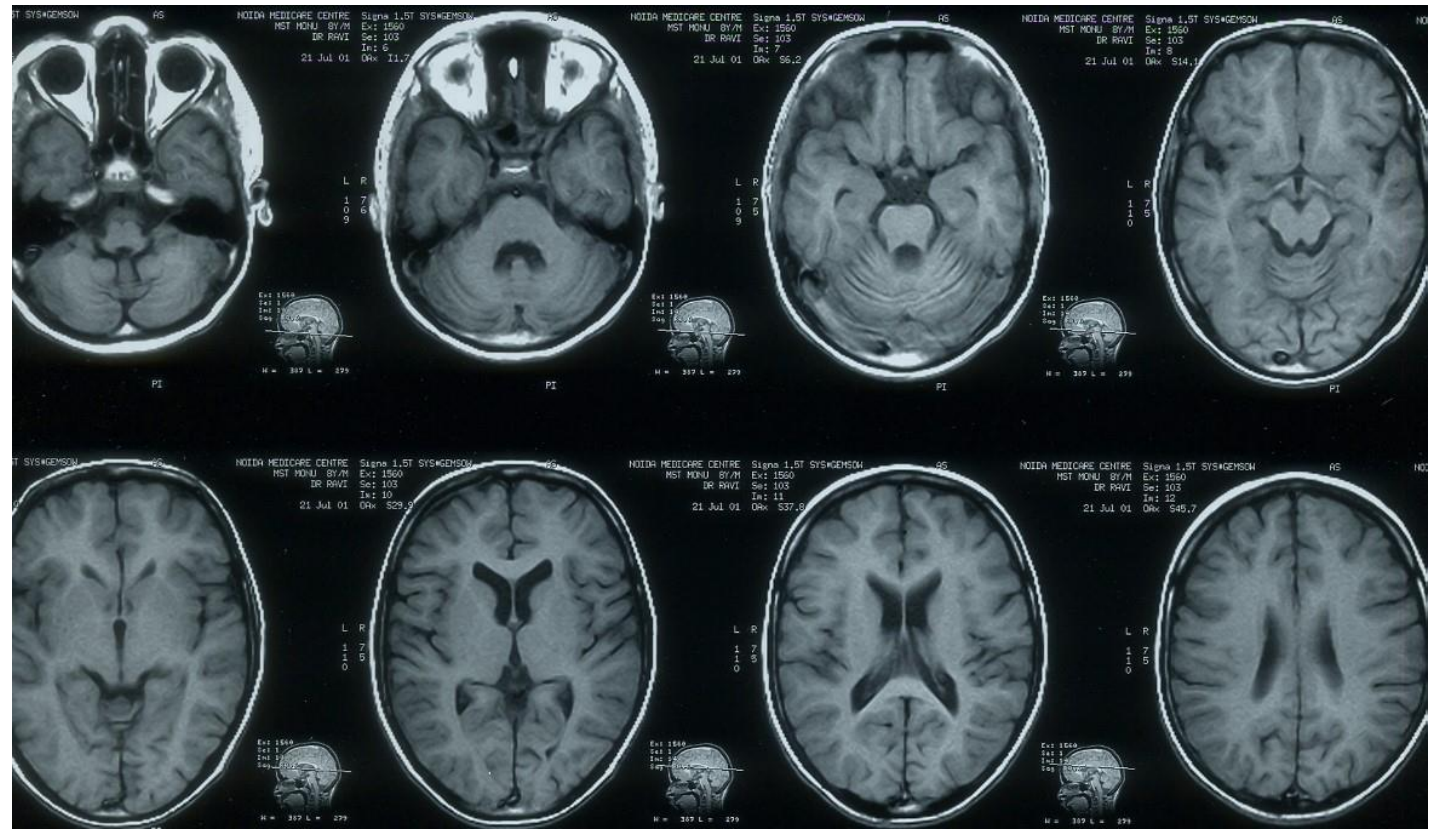

Fig. 2. Normal brain MRI of the patient at the age of 7 years

At age of 11 years brain MRI showed cerebral and cerebellar atrophy (Figure 3). Repeated brain MRI in the present admission revealed diffuse cerebral and cerebellar atrophy with hypodensity in thalamus and few white matter changes in bilateral frontal area suggestive of a neurodegenerative disorder (Figure 4).

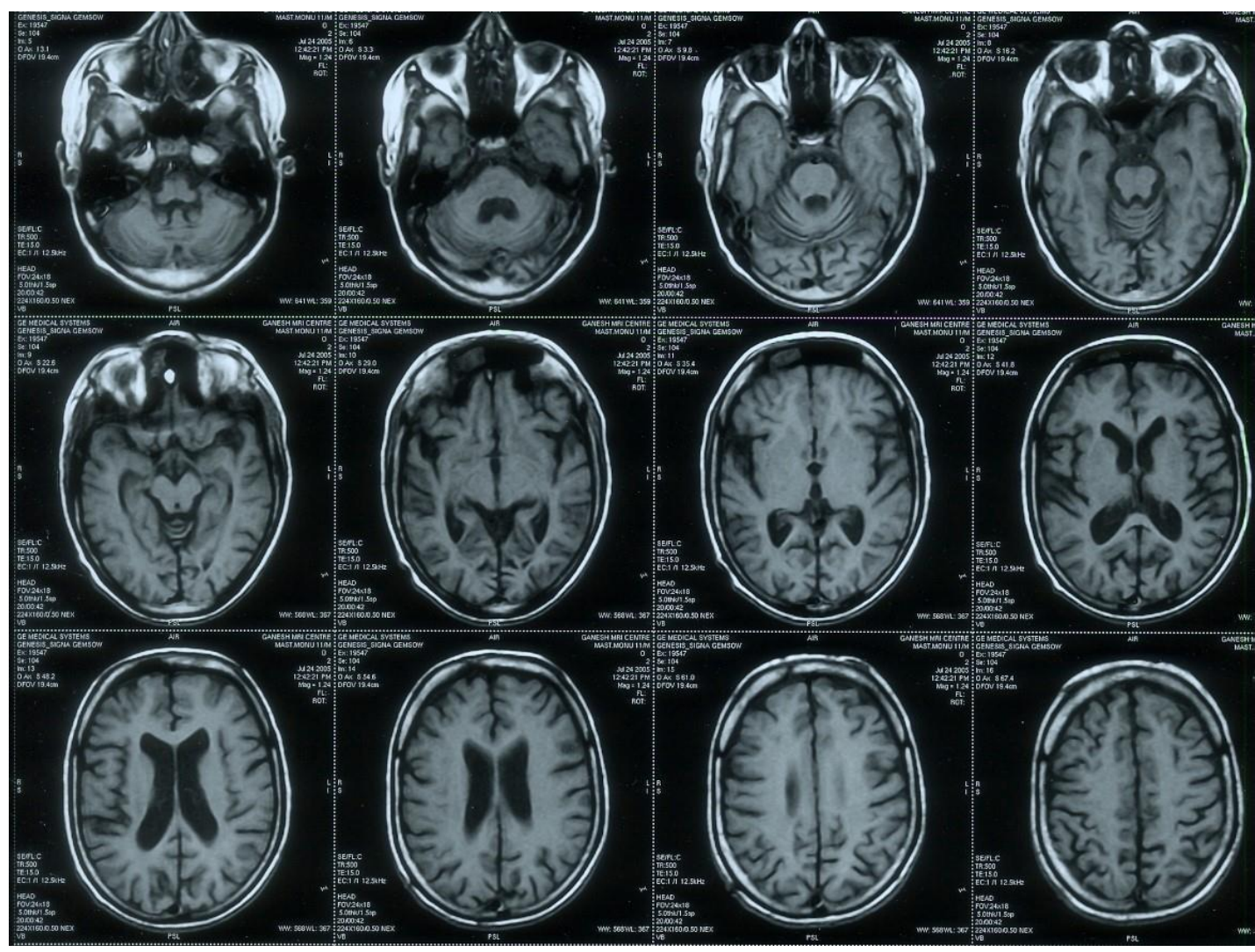

Fig. 3. Brain MRI showed cerebral and cerebellar atrophy at age of 11 years 


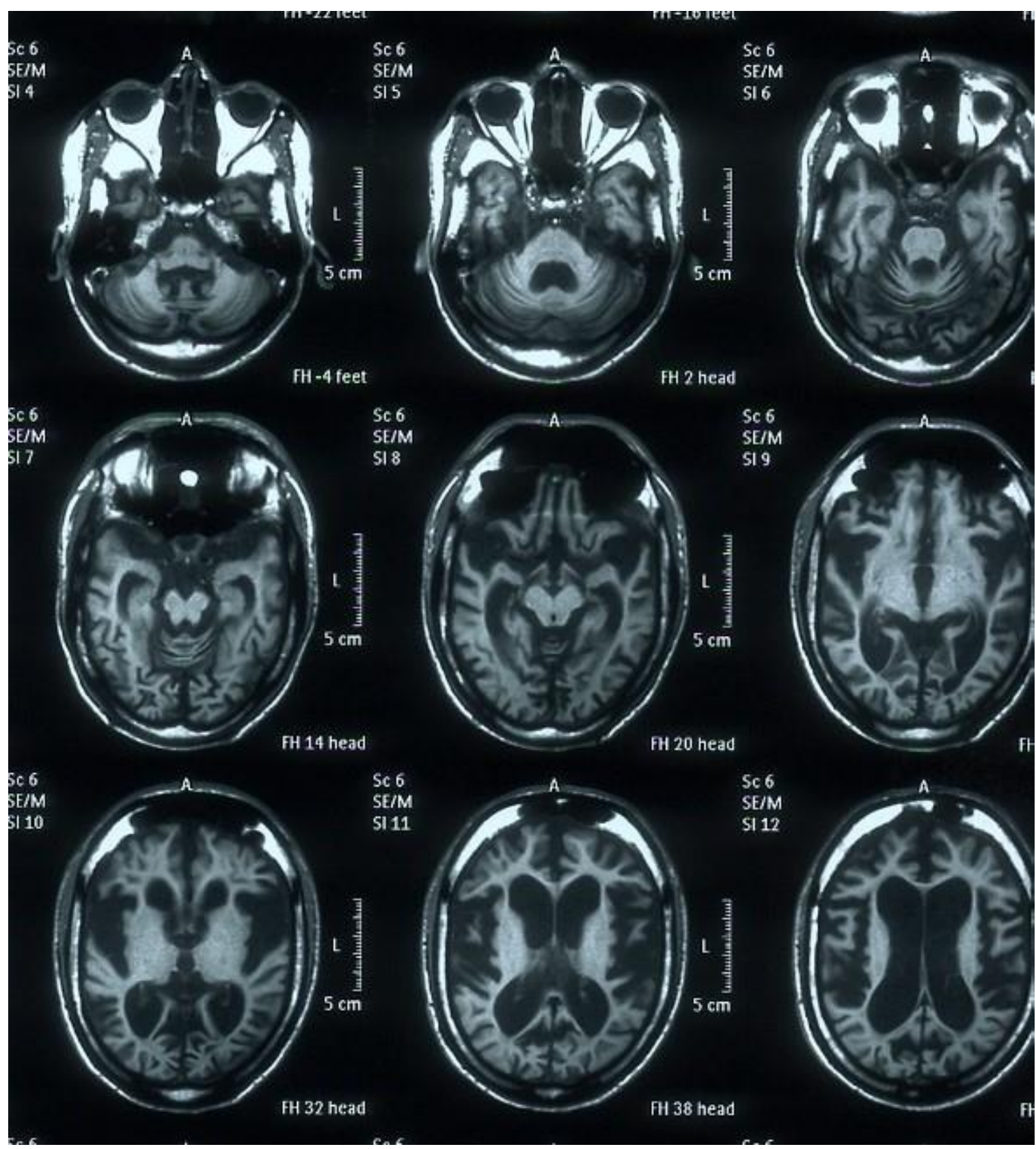

Fig. 4. Brain MRI at age of 21 years revealed diffuse cerebral and cerebellar atrophy with hypodensity in thalamus and few white matter changes in bilateral frontal area suggestive of a neurodegenerative disorder

Based on clinical history of seizures, behavioral disturbances and rapid deterioration of vision resulting in blindness with classical funds changes and collaborating MRI changes in background of similar complaints in elder brother with fatal outcome, diagnosis of Batten disease was considered.

Specialized tests including microscopic examination and histological examination of tissue sample of skin biopsy, electroretinography and visual evoked potential were planned but could not be done because of non-availability and financial constraints of the patient.

Patient was started on sodium valproate and clobazam and seizures were controlled. $\mathrm{He}$ was discharged after a week of observation and is being followed up routinely. Genetic studies could not be done due to nonavailability of tests in our institute and nonaffordability of patient's family for outside investigations. 


\section{Discussions}

Batten disease is an autosomal recessive disease. It occurs due to disruptions or mutations of the CLN3 gene located on the short arm (p) of chromosome 16 (16p12.1) [2, $3]$. The gene codes for a protein called battenin, which is found in the membranes of the cell (most predominantly in lysosomes and in related structures called endosomes). It affects males and females in equal numbers. It is often misdiagnosed as retinitis pigmentosa, macular degeneration, mental retardation, epilepsy, Attention Deficient Hyperactivity Disorder, autism and, even, schizophrenia.

Diseases like GM1 gangliosidosis, Alpers disease, idiopathic generalized epilepsy and other forms of NCL are close differential diagnosis of this disease. However patients with Infantile GM1 gangliosidosis, have seizures along with hepatosplenomegaly, skeletal abnormalities, seizures, profound intellectual disability, and show a characteristic cherry red spot in retina and usually do not survive past early childhood. Patients with Alpers' disease show a triad of seizures, psychomotor regression, and liver disease. Idiopathic generalized epilepsy (IGE) constituting about one third of all epilepsies, is a group of epileptic disorders having a genetic basis, a positive family history and having no structural brain abnormalities. Apart from Batten disease, NCL disorders mainly have other four subtypes, three forms beginning earlier in childhood and a rare form that affects adults. They have characteristic genetic mutations which become apparent at different ages and show almost similar symptoms like Batten disease.

Neuropathology in Batten disease is marked by two major features: neuronal degeneration and accumulation of intracellular lipopigments. Neuronal degeneration begins in the dendritic tree leading to neuronal loss predominating in cortical regions of cerebrum and cerebellum. Lipopigments are found inside lysosomes and consist of fats and proteins. They largely accumulate in the perikarya of nerves and glial cells and are found in cells of the brain and the eye as well as in skin, muscle, and many other tissues. The accumulated lipopigments form distinctive shapes that can be seen under an electron microscope.

In the beginning, these children reach usual developmental milestones on schedule. The most common initial symptom is the slow gradual decline of vision or seizures. As the illness progresses, there is loss of central vision and color blindness followed by loss of night vision. Eventually peripheral sight is lost finally resulting in total blindness. The first epileptic seizure is noticed at the mean age of 10 years [4]. The seizures may be either petit mal (absence) or grand mal (tonic-clonic). In addition to petit mal and grand mal seizures, these children may also suffer from drop seizures (atonic), psychomotor (complex partial or temporal lobe), myoclonic, and Jacksonian (simple partial) type of seizures. Patients also suffer from a number of different psychiatric symptoms. The most commonly reported symptoms are social, thought, and attention problems, somatic complaints and aggressive behavior. They also have repetitive speech disorder known as echolalia and eventually lose their speech totally. Extrapyramidal symptoms are noticed in about half of the patients between the ages of 12 and 15 years, and in the rest later [5]. Extrapyramidal signs include impaired balance, rigidity, hypokinesia, stooped posture and shuffling gait. As the disease progresses motor skills become more affected. During the late teens or twenties physical abnormalities develop including sudden involuntary muscle contractions (myoclonus), muscle spasms (spasticity) that result in slow, stiff movements of the legs, and weakness or paralysis of all four limbs (quadriparesis). This eventually leads to the loss of mobility progressing on to a bed ridden stage. In patients with the homozygous form of the major mutation, the disease leads to premature death at a mean age of 24 years.

There are various hematological, radiological and histopathological investigations which supports the diagnosis of Batten disease, although the definite diagnosis rests upon genetic studies. The presence of vacuolated lymphocytes i.e. white blood cells that contain holes or cavities (observed by microscopic analysis of blood smears), when combined with other findings that indicate 
$\mathrm{NCL}$, is suggestive for the juvenile form caused by CLN3 mutations. On electron microscopy skin, muscle, rectal and conjunctiva tissue samples reveal abnormal accumulations of lipofuscin deposits in the form of inclusion bodies within several different types of cells. These inclusion bodies are normally seen in the form of "fingerprints" and look exactly as the name implies. Secretory eccrine sweat gland epithelial cell is the most important cell for histological examination and therefore a punch biopsy deep enough to include sweat glands is necessary.

Ophthalmoscopically, there is an early bull's-eye maculopathy, but the entire retina is affected early, as evidence by full-field electroretinography changes. Early on, there is characteristic attenuation of the $b$ wave on electroretinogram with a normal electrooculogram. Later, there is widespread photoreceptor degeneration with bone spicules, vascular attenuation, salt and pepper macula, bull's eye lesion and optic atrophy. In the electroencephalogram, progressive background abnormality and an increase in paroxysmal activity are seen [6]. Electroretinography (ERG) and visually evoked potential (VEP) are other tests which can aid in the diagnosis of juvenile Batten disease. ERG shows lack of response when the eye is stimulated by light, due to optic nerve abnormalities thus confirming progressive retinal pigmentary degeneration. Markedly reduced b:a ratio in the single flash photopic ERG with additional a-wave delay is the most suggestive feature of Batten disease [7]. Brain MRI is usually normal under the age

\section{References}

1. Batten FE. Cerebral degeneration with symmetrical changes in the maculae in two members of a family. Transactions of the Ophthalmological Societies of the United Kingdom 1902; 23:386-390.

2. Hobert JA, Dawson G. Neuronal ceroid lipofuscinoses therapeutic strategies: past, present and future. Biochim Biophys Acta 2006; 1762(10):945-953.

3. Rakheja D, Narayan SB, Bennett MJ. Juvenile neuronal ceroid-lipofuscinosis (Batten disease): of 10 years. After the age of 10 years, variable cerebral and cerebellar atrophy appears. In addition, the thalami may show abnormally low signal intensity and the periventricular white matter high signal intensity on T2-weighted images from the age of 11 years [8]. In families where the mutation in the gene for CLN3 is known, DNA analysis can be used to confirm the diagnosis or for the prenatal diagnosis of Batten disease. When the mutation is known, DNA analysis can also be used to detect unaffected carriers of this condition for genetic counseling.

\section{Conclusion}

Refractory seizures along with behavioural disturbances and rapid deterioration of vision in young patients, although rare, diagnosis of Batten disease should be considered. Classical funds changes, collaborating MRI changes and Genetic testing help to establish the diagnosis. Early diagnosis is important as it is a progressive disease and result in early mortality.

\section{Consent}

Written informed consent was obtained from the patient for publication of this case report and accompanying images.

\section{Conflict of interest}

The author(s) declare that they have no competing interests.

a brief review and update. Cure Mol Med 2007; 7(6):603-608.

4. Aberg L, Bäckman M, Kirveskari E, Santavuori P. Epilepsy and antiepileptic drug therapy in juvenile neuronal ceroid lipofuscinosis. Epilepsia 2000; 41:1296-1302.

5. Jarvela I, Autti T, Lamminranta S, Aberg L, Raininko R, Santavuori P. Clinical and magnetic resonance imaging findings in Batten disease:analysis of the major mutation $(1.02 \mathrm{~kb}$ deletion). Ann Neurol 1997; 42:799-802. 
6. Larsen A, Sainio K, Aberg L, Santavuori P. Electroencephalography in juvenile neuronal ceroid lipofuscinosis: visual and quantitative analysis. Eur J Paediatr Neurol 2001; 5(1):179183.

7. Collins J, Holder GE, Herbert H, Adams GGW. Batten disease: features to facilitate early diagnosis. Br J Ophthalmol 2006; 90(9):11191124.

8. Autti $T$, Raininko R, Vanhanen $S L$, Santavuori P. MRI of neuronal ceroid lipofuscinosis. Part I: cranial MRI of 30 patients with juvenile neuronal ceroid lipofuscinosis. Neuroradiology 1996; 38(5):476-482. 